(c) 2010 IEEE. Personal use of this material is permitted. Permission from IEEE must be obtained for all other uses, in any current or future media, including reprinting/republishing this material for advertising or promotional purposes, creating new collective works, for resale or redistribution to servers or lists, or reuse of any copyrighted component of this work in other works. 


\title{
Subjective Quality Assessments in Virtual Environments
}

\author{
Azadeh Rajabian Tabesh, Farookh Khadeer Hussain, Elizabeth Chang, Omid Ameri Sianki \\ Digital Ecosystems and Business Intelligence (DEBI) Institute \\ Curtin Business School \\ Curtin University of Technology \\ Perth, Australia \\ \{A.RajabianTabesh, F.Hussain, E.Chang\}@curtin.edu.au
}

\begin{abstract}
Being able to make objective Quality of Service (QoS) judgments or assessments is a challenging and crucial activity. The process of making these assessments is compounded when the environment in which the assessments have to be made are virtual; in the sense the interacting parties might not have necessarily met with each other physically. In a broad sense Quality of Service assessments could be broadly categorized into two areas, namely objective assessments and subjective assessments. In this paper, we propose a suite of metrics to carry out subjective quality assessment in a virtual environment.
\end{abstract}

Keywords: Trust, Trustworthiness, Quality Assessments, Subjective Assessment, Objective Assessment.

\section{INTRODUCTION}

Trust and reputation technologies are key in enabling a service requestor to make Quality of Service (QoS) assessments. Due to this reason there has been a lot of research in the past few years on trust and reputation as a technology as well as a means to make QoS or QoP judgments [1, 3, 9 and 10]. Chang and Hussain et al [3, 4 and 7] propose a suite of metrics, which they term as CCCI metrics in order to make objective QoS or QoP assessments. The working of their proposed methodology is based on both the interacting parties agreeing upon a set of criteria, prior to the interaction, which subsequently form the basis for assessment.

However, in some scenarios, there may not necessarily be a set of guiding or mutually agreed criteria or factors based on which the assessment process would be carried out eventually. In contrast to an objective assessment process, as proposed by Chang and Hussain et al [3, 4 and 7], the assessment criteria in such a scenario could be decided by the trusting agent (service requestor) with little or no input or agreement from the other interacting party (trusted agent or service provider). We term such a scenario where the assessment criteria or factors have been decided without the mutual agreement of both the interacting parties as subjective assessment. This is in contrast to the scenario where in the assessment criteria have been agreed mutually by both the interacting parties. Since the assessment criteria have been agreed to by both the interacting parties, we term such an assessment process as objective assessment.

Researchers have investigated the use of objective trust assessment metrics for objective trust assessment (CCCI metrics) in the literature. However subjective trust assessment has received little or no attention thus far in the literature. In this paper, we propose a suite of metrics which can be used for subjective trust assessment in contrast to the CCCI metrics which are used to quantify and express objective trust assessment value. The suite of metrics, which we propose in this paper are based on the CCCI metrics. Interested readers may refer to [4, 7] for a thorough discussion about CCCI metrics with examples.

The remainder of this paper is organized as follows: In Section 2, we present an overview of the existing definitions of trust in the literature. Additionally, we draw clear boundaries' between objective trust and subjective trust. In Section 3, we propose a scale for capturing and representing the subjective nature of trust. It is important to note that the proposed scale for measuring subjective trust is in line with the scale proposed for measuring of objective trust. In Section 4 , we define a suite of metrics for measuring and expressing subjective trust, both quantitatively and qualitatively. Finally Section 5 concludes the paper and sets the ground for further research.

\section{AN OVERVIEW OF TRUST DEFINITIONS IN THE EXISTING LITERATURE}

Trust has been defined in various ways by researchers. These variations in the definition of trust arise from the domain specific interpretation of trust. Due to space constraints it is not possible to present an overview of all the existing definitions of trust in the existing literature. In this 
paper, we focus our attention only on the most popularly cited definitions of trust in the existing literature. Interested readers are requested to refer to Hussain et al [9] for an indepth discussion and thorough evaluation of all the proposed definitions of trust by researchers from various fields.

Josang et al [1] define trust in a passionate entity as "the belief that it will be behaved without any malicious intent". Additionally, Jasong et al define trust in a rational entity as "the belief that it will resist malicious manipulation by a passionate entity". Josang et al's definition of trust does not take into consideration the subjective nature of trust. Additionally, they do not draw the differences between the two broad forms of trust.

Mui et al [5,6] define trust as "a subjective belief an agent has about another's future behaviour based on history of their encounters". The definition of trust by Mui et al takes into account the subjective nature of trust. However, it does not take into account the fact that trust is a reflection of the other interacting parties willingness and capability. Additionally their definition fails to define subjective trust as being a function time and context.

Gambetta et al [2] define trust as "trust is a particular level of the subjective probability with which an agent will perform a particular action, both before we can monitor such action <or independently of his capacity of ever to be able to monitor it $>$ and in a context in which it affects our own action". Similar to the definition of trust by Mui et al, this definition takes into account the subjective nature of trust. However, similar to Mui et al, it does not take into account the fact that trust is a reflection of the other interacting parties willingness and capability. Additionally their definition fails to define subjective trust as being a function time.

Buist et al [8], define trust as "to trust is to willingly relinquish control, making yourself vulnerable to someone else for a certain outcome or consequence. Trust grows as a result of positive experiences accumulated over time". Similar to the definition of trust by Josang et al, this definition of trust does not take into consideration the subjective nature or objective nature of trust.

Hussain et al [4,7], define trust as "the belief that the trusting agent has in the trusted agents willingness and capability to act in a mutually agreed manner in a given context and at a given point in time". The definition of trust by Hussain et al takes into account the context-dependent and time-dependent nature of trust. Additionally, it takes into account that trust is a reflection of the other interacting parties willingness and capability. However the above definition of trust proposed by Hussain et al is focussed only on objective trust. This is due to the fact that their definition states that trust results due to the mutually agreed criteria between the interacting parties. It does not take into account that trust could be a subjective assessment by the trusting agent about the trusted agent.

As we can see from the above discussion, some of the existing definitions of trust focus on trust being a subjective asssessment by the trusting agent. However, they fail to take into account that this subjective assessment would be a function of both context and time. Additionally, they fail to consider that this subjective assessment would be reflection of the willingness and capability of the other interacting party.

Another major shorcoming with the existing literature is that it fails to propose a framework or a methodology by which subjective trust could be measured or expressed either quantitatively or qualitatively. In this paper we will propose a suite of metrics, by which the trusting agent can express both quantitaively and qualitatively its subjective trust assessment. However in order to do that, we need to define quantitative and qualitative measures to express the subjective trust value.

In the next section, we propose our definition of subjective trust which addresses the shortcomings discussed above. Additionally, we propose a scale for measuring and expressing subjective trust value. This scale is in line with the scale proposed by Hussain et al [4,7] for measuring objective trust.

In Section IV, we propose a set of metrics of for measuring subjective trust. In Section V, the working of these metrics is illustrated by making use of a case study. Section VI concludes this paper and outlines the scope for future research.

\section{Definition Of SubJective Trust}

We define subjective trust as "the belief that the trusting agent has in the trusted agents' capability and willingness to act as expected (by the trusting agent) in a given context at a given time".

The terms 'trusting agent', 'belief', 'trusted agent', 'capability', 'willingness', 'context' and 'time' have the same semantics as that for objective trust. Interested readers are requested to refer to Hussain et al $[3,9]$ for an indepth discussion of these terms. The major difference in the definition of subjective trust is the use of the words 'to act as expected' in contrast to the words 'to act in a mutually agreed manner' for objective trust.

As mentioned previously in objective trust and consequently for objective trust assessments, both the interacting parties, agree on a collection of criteria based on which the assessment process would be carried out. However, in contrast to objective trust and objective trust assessments, in subjective trust assessments, the evaluation process is carried out against a set of criteria which have not been agreed between the interacting parties.

In line with the levels of trust proposed by Chang and Hussain et al $[4,7]$, we propose seven different levels of subjective trust. These trust levels are shown below in Table 1. 
TABLE I. LEVELS OF SUBJECTIVE TRUSTWORTHINESS

\begin{tabular}{|c|c|c|}
\hline $\begin{array}{c}\text { Trustworthiness } \\
\text { level } \\
\text { Quantitative } \\
\text { Representation) }\end{array}$ & $\begin{array}{c}\text { Trustworthiness } \\
\text { Value }\end{array}$ & $\begin{array}{c}\text { Quantitative } \\
\text { Representation }\end{array}$ \\
\hline Level 0 & $\mathrm{X}=$ Not defined & Unknown Agent \\
\hline Level 1 & $0<\mathrm{X} \leq 1$ & $\begin{array}{c}\text { Completely } \\
\text { Untrustworthy }\end{array}$ \\
\hline Level 2 & $1<\mathrm{X} \leq 2$ & Untrustworthy \\
\hline Level 3 & $2<\mathrm{X} \leq 3$ & Minimally Trustworthy \\
\hline Level 4 & $3<\mathrm{X} \leq 4$ & Partially Trustworthy \\
\hline Level 5 & $4<\mathrm{X} \leq 5$ & Trustworthy \\
\hline Level 6 & $5<\mathrm{X} \leq 6$ & $\begin{array}{c}\text { Completely } \\
\text { Trustworthy }\end{array}$ \\
\hline
\end{tabular}

\section{OVERVIEW OF METRICS FOR SUBJECTIVE TRUST}

In this section, we present and define the metrics, using which the trusting agent (service requestor) can carry out subjective quality assessment about the trusted agent (service provider). The metrics we make use of are as follows:

1. Correlation of an Interaction (Corr Interaction $_{\text {) }}$ In the case of subjective trust assessments, this metric represents the degree of consonance between the actual service (provided by the service provider) and the desired service (from the perspective of service requestor).

Note that in objective trust assessment, the benchmark for comparing the actual service would be the mutually agreed service. In contrast, for subjective trust assessments, the benchmark is the desired service from the perspective of the service requestor.

2. Correlation of Criteria $\left(\operatorname{Corr}_{\text {Criteria }}\right)$ : In the case of subjective trust assessments, this metric represents the degree of consonance between the actual behavior of the service provider in a given criteria and the desired behavior in that criterion (from the perspective of service requestor).

In objective trust assessments, the benchmark for comparing the actual service for that criterion would be the mutually agreed service for that criterion. In contrast, for subjective trust assessments, the benchmark is the desired service for that criterion from the perspective of the service requestor.
3. Importance of Criteria ( $\operatorname{Imp}_{\text {Criteria): This metric captures }}$ the importance of a given criteria in an overall interaction, as perceived by the service requestor.

In the case of measuring subjective trust, the correlation

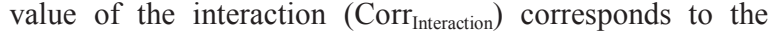
subjective trustworthiness of the service provider.

In order to have the importance of a criterion (Imp criterion), have an effect on the correlation value of the corresponding criterion $\left(\right.$ Corr $\left._{\text {criterion }}\right)$, we multiply the Corr criterion value by $\mathrm{Imp}_{\text {criterion. }}$ Similar to the case of objective trust assessments, the importance of criteria can take one of the following numerical values: 0,1 or 2 .

The service requestor can assign a value of 0 to Imp criterion, which would mean that the criterion is unimportant in determining the outcome of the interaction. Alternatively, the service requestor can assign a value of 1

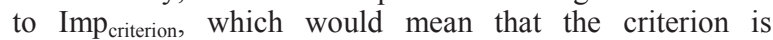
important in determining the outcome of the interaction. Finally, the service requestor can assign a value of 2 to Imp criterion, which would mean that the criterion is extremely important in determining the outcome of the interaction.

Assuming there are ' $\mathrm{m}$ ' criteria in the interaction, the actual behavior (or actual service provided by the service provider), denoted as Service $_{\text {Actual, }}$ of an interaction is a function of the correlation value of each individual criterion weighted by the corresponding importance of the criterion. It is important to note that unlike objective trust assessments, in subjective trust assessment the clarity of each individual criterion is not taken into account. The actual behavior of the of the service provider, Service ${ }_{\text {Actual }}$, can be mathematically formulated as follows:

$$
\sum_{\mathrm{i}=1}^{\mathrm{m}} \mathrm{f}(\text { Corr Criteria i } * \text { Imp Criteria i })
$$

In order to determine the subjective trustworthiness value of the interaction, we would have to compare the actual behavior or service provided by the service provider with the desired service (from the perspective of the service requestor). Assuming there are ' $n$ ' criteria in the desired service, the desired behavior (or desired service from the perspective of service requestor), denoted as Service ${ }_{\text {Desired, }}$ can be mathematically formulated as follows:

$$
\sum_{j=1}^{n} f(\text { Corr Criteria j * Imp Criteria j })
$$


The correlation of an interaction would then be represented as follows:

$$
\text { Corr }_{\text {Interaction }}=\text { Service }_{\text {Actual }} / \text { Service }_{\text {Desired }}
$$

Making use of (1) and (2) in (3) we can determine the subjective trustworthiness of a service provider as follows:

$$
\text { Subjective trustwor thiness }=6 * \frac{\sum_{\mathrm{i}=1}^{\mathrm{m}}\left(\operatorname{Corr} \text { Criteria }_{\mathrm{i}} * \operatorname{Imp} \text { Criteria } \mathrm{i}\right)}{\sum_{\mathrm{j}=1}^{\mathrm{n}}\left(\operatorname{Corr} \text { Criteria }_{\mathrm{j}} * \operatorname{Imp} \text { Criteria } \mathrm{j}\right)}
$$

The service requestor can make use of (4) above in order to determine the subjective trustworthiness value of the service provider. In most cases the desired service from the service requestor would be the same as the state of art service as experienced by the service requestor in the same industry in the past.

However, if the purpose of the trust assessment is to determine the objective trustworthiness value then the actual service needs to be compared against the mutually agreed service as shown in (5) below.

\section{Objective trustworthiness value $=$}

$$
\text { Corr }_{\text {Interaction }}=\text { Service }_{\text {Actual }} / \text { Service }_{\text {MutuallyAgreedService }}
$$

\section{CASE STUdY OF CARRYING OUT SUBJECTIVE TRUST ASSESSMENT}

In this section, we with the help of a case study, we intend to illustrate the process of determining the subjective trustworthiness value of a service provider. We additionally wish to illustrate and point out using the following case study that for the same service provided by a given service provider, the subjective quality assessments may not necessarily be the same, by different service requestors.

Let us consider that Mary and Jack have both stayed in Maxim Hotel for a duration of three days each. During their stay at the Maxim Hotel, they were provided with service comprising of the following criteria: (a) Hotel room; (b) Gym Facility; (c) 24*7 Internet Facility; and (d) Room Service

Let us assume that Mary's expectations of service from the service provider (Maxim Hotel) were as follows: (a) A reasonably sized hotel room; (b) Gym Facility; (c) 24*7 Internet Facility; (d) 24*7 Room Service; (e) A tour of the city; and (f) A range of cuisine including at least international cuisine. It is important to note here that Mary's expectation of the above service could be due to similar previous service received by Mary in the same domain or industry in the past. Let us assume further than Mary regards all the above criteria as important in determining the successful outcome of the interaction.

On the other hand, let us assume that Jack's expectations of service from the same service provider (which could be due Jack's domain specific state-of-art experienced service was as follows: (a) A reasonably sized hotel room; (b) $24 * 7$ Room Service. Let us assume furthermore for discussion purposes that Jack regards both of the above criteria as very important in determining the successful outcome of the interaction.

Let us first determine the (subjective) trust value that Mary would have after interaction with Maxim hotel.

TABLE II. TABLE DEPECTING MARY'S ASSESSMENT OF THE CRITERIA AND THEIR IMPORTANCE

\begin{tabular}{|c|c|c|}
\hline Criterion Name & $\begin{array}{c}\text { Correlation of } \\
\text { Criterion from } \\
\text { Mary's Perspective }\end{array}$ & $\begin{array}{c}\text { Importance from } \\
\text { Mary's Perspective }\end{array}$ \\
\hline Hotel Room & 1 & 1 \\
\hline Gym Facility & 1 & 1 \\
\hline Internet Facility & 1 & 1 \\
\hline Room Service & 1 & 1 \\
\hline Tour Facility & 0 & \\
\hline Selection/Variety & 0 & \\
\hline
\end{tabular}

Assuming that the provided hotel room, gym facility, internet facility and room service facility is in line with Mary's requirements, Mary's subjective trustworthiness value for Maxim Hotel can be calculated, by making use of (4), as follows:

Mary's Subjective Trustworthiness Value $=$

$6^{*}\left(\left(1^{*} 1\right)+\left(1^{*} 1\right)+\left(1^{*} 1\right)+(1 * 1)+\left(0^{*} 1\right)+\left(0^{* 1}\right)\right) /\left(\left(1^{*} 1\right)+\left(1^{*} 1\right)+\left(1^{*} 1\right)+\left(1^{* 1}\right)+\left(0^{*} 1\right)+\left(0^{*} 1\right)\right)$

Mary's Subjective Trustworthiness Value $=4$

In qualitative terms or semantically, this would correspond to Maxim Hotel being partially trustworthy from Mary's perspective (from Table I). It is important to note that in the same scenario an objective trustworthiness value for Maxim hotel would be 6 , semantically corresponding Maxim Hotel being completely trustworthy. 
Let us now look at the (subjective) trust value that Jack would have after interaction with Maxim hotel.

TABLE III. TABLE DePeCting JACK'S ASSESSMENT OF THE CRITERIA AND THEIR IMPORTANCE

\begin{tabular}{|c|c|c|}
\hline Criterion Name & $\begin{array}{c}\text { Correlation of } \\
\text { Criterion from } \\
\text { Jack's Perspective }\end{array}$ & $\begin{array}{c}\text { Importance from } \\
\text { Jack's Perspective }\end{array}$ \\
\hline Hotel Room & 1 & 2 \\
\hline Internet Facility & 1 & 2 \\
\hline
\end{tabular}

Jack's subjective trustworthiness value for Maxim Hotel can be calculated, by making use of (4), as follows:

Jack's Subjective Trustworthiness Value =

$$
6 *((1 * 2)+(1 * 2) /(1 * 2)+(1 * 2))
$$

\section{Jack's Subjective Trustworthiness Value $=6$}

In qualitative terms or semantically, this would correspond to Maxim Hotel being completely trustworthy from Jack's perspective (from Table I).

As we can see from the above case study, for the same service provided by Maxim hotel, two different service requestors' (Jack and Mary) have different perceptions of the provided service. The difference in their perception for the provided service could be due to the difference in their experienced state of the art service previously in the same domain or industry. For example, Mary might be used to staying in hotels wherein the criteria expected from her (as laid out in the case study) could be the norm. Hence, for Mary the quality of service provided by Maxim Hotel can be regarded as partially trustworthy.

On the other hand, Jack could be used to staying in hotels where in the desired criteria by Jack could be the norm. Hence, Jack's subjective assessment the quality of service provided by Maxim Hotel can be regarded as completely trustworthy.

\section{CONCLUSION AND FUTURE WORK}

In this article we introduced and defined the notion of subjective trust. We made a distinction between subjective trust assessments and objective trust assessment. Subsequently, based on the CCCI metrics, we proposed a suite of metrics for carrying out subjective trust assessment and expressing the outcome of such an assessment process, both qualitatively and quantitatively.

Finally, we made use of a case study to illustrate the process of carrying out subjective trust assessments. It was noted that for the same service, different service requestors may assign different trustworthiness values. This difference could be primarily due to the difference in perception of the service arising from the previous experienced state of the art service.

Our further work involves making use of the above methodology in a real working environment. Additionally, we intend to investigate the scenarios in which it may be optimal to use subjective trust assessment and objective trust assessment.

\section{REFERENCES}

[1] Audun Josang, "The Right Type of Trust for Distributed Systems", Proceedings of the 1996 workshop on new security paradigms, Lake Arrowhead, USA, 16-19 September 1996, pp.119-131.

[2] Deigo Gambetta, "Can we trust trust?" Trust: Making and Breaking Cooperative Relations, Basil Blackwell, New York, pp. 213-237.

[3] E.Chang, F.Hussain, T.Dillon, 'Trust and Reputation For ServiceOriented Environments”, ,vol.1, John Wiley \& Sons (2006).

[4] Farookh Khadeer Hussain, Elizabeth Chang, Tharam Dillon, "Trustworthiness and CCCI metrics in P2P communication", Perth, Australia, 2007.

[5] Lik Mui, Ari Halberstadt, Mojdeh Mohtashemi, "Evaluating Reputation in Multi-agents Systems", Proceedings of the International Workshop on Trust, Reputation, and Security: Theories and Practice, Bologna, Italy, 15 July 2002, pp. 123-137.

[6] Lik Mui, Mojdeh Mohtashemi, Ari Halberstadt, "Notions of reputation in multi-agents systems: a review", Proceedings of the First International Joint Conference on Autonomous Agents and Multi-Agent System (AAMAS 2002), Bologna, Italy, 15-19 July 2002, pp. 280-287.

[7] Farookh Khadeer Hussain, "A Methodology for Trust Modelling in Service Oriented Environments", PhD Thesis, Curtin University of Technology, 2007, 450 pages.

[8] Ken Buist, "Definition of Trust", Available: http://www.teamtechnology.co.uk/trustworthiness/definition-oftrust.html, Retrieved: 05/06/2010.

[9] Mohammad Gias Uddin, M. Z., Sheikh Iqbal Ahamed (2008). CAT: a context-aware trust model for open and dynamic systems. Fortaleza, Ceara, Brazil, ACM New York, NY, USA: 2024-2029.

[10] Wen-jing Cui, Hai-yang Wang, Qi Sui, and Li-zhen Cui , "Trust Management Model and Evaluation Method forOnline Travel",School of Computer Science and Technology, Shandong University, China, Volume 5236/2008 